\title{
Optics for the Antiproton Decelerator at CERN
}

\author{
P. Beloshitsky, C. Carli, S. Maury, D. Möhl, F. Pedersen \\ CERN, Geneva, Switzerland
}

\begin{abstract}
The former Antiproton Collector (AC) at CERN has been transformed into an Antiproton Decelerator (AD) to supply high quality antiproton beams with a momentum of $100 \mathrm{MeV} / \mathrm{c}$ (kinetic energy of $5.3 \mathrm{MeV}$ ) [1]. As in AC, antiprotons are injected at $3.57 \mathrm{GeV} / \mathrm{c}$ and cooled by stochastic cooling system. To facilitate the deceleration and to prepare a small emittance beam for the experiments, stochastic cooling at $2 \mathrm{GeV} / \mathrm{c}$ and electron cooling at $300 \mathrm{MeV} / \mathrm{c}$ and $100 \mathrm{MeV} / \mathrm{c}$ has been incorporated. The required optics modifications are described and the results of machine commissioning are discussed.
\end{abstract}

\section{INTRODUCTION}

The AC optics was mainly determined by the requirements for stochastic cooling: a) machine operation close to transition energy and b) betatron phase advances between pick-ups and kickers of the stochastic cooling system close to $90^{\circ}+180^{\circ} \times \mathrm{n}$ ( $\mathrm{n}$ is integer). Four quadrupole families were used to control the machine tunes $\mathrm{Q}_{x}, \mathrm{Q}_{y}$ and the dispersion and its derivative $\mathrm{D}_{x}, \mathrm{D}_{x}^{\prime}$. The magnets, vacuum chambers and all other equipment were designed to have an acceptance of $240 \pi \mathrm{mm}$ mrad in both planes with the lattice functions obtained.

While $\mathrm{AC}$ operated at fixed momentum, in $\mathrm{AD}$ during deceleration a momentum is reduced by factor of 36 . To compensate the adiabatic emittance increase, the electron cooling at low momenta has been incorporated. The electron cooler is located in one of the 4 straight sections, where a special insertion was created by rearranging adjacent quadrupoles [2].

\section{AD OPTICS DESIGN}

With this new layout, it was impossible to keeps $\mathrm{AC}$ tunes $\mathrm{Q}_{x}=5.46, \mathrm{Q}_{y}=5.43$ due to insufficient strength in the quadrupoles around the e-cooler. The new working point near the main diagonal $\mathrm{Q}_{x}=5.39, \mathrm{Q}_{y}=5.37$ has been proposed to provide sufficient room free from the low order resonances (see Fig. 1). The number of independent quadrupole families was increased from 4 to 6 to control the beta functions in the e-cooler in addition to other constraints mentioned above.

With aperture limitations coming from AC machine acceptances as defined by linear optics at injection energy are reduced to $\mathrm{A}_{x}=224 \pi \mathrm{mm} \mathrm{mrad}$ and $\mathrm{A}_{y}=192 \pi \mathrm{mm} \mathrm{mrad}$. Further reduction comes from non-linearities. The AD has no sextupole lenses to compensate the chromaticity of quadrupoles, but sextupole components are created by special pole profile in the quadrupoles located in dispersion

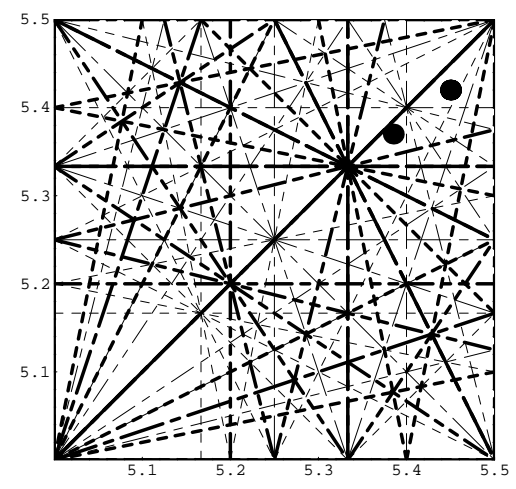

Figure 1: $\mathrm{AD}$ tune diagram $\left(\mathrm{Q}_{x}, \mathrm{Q}_{y}\right)$ with two working points now in operation. Resonances up to 6 order are shown.

regions. To reduce non-linearities in the $\mathrm{AC}$ [3], sextupole windings in the 6 narrow quadrupoles (placed in dispersion free straight sections) were used. In $\mathrm{AD}$, this compensation procedure has been employed again, and sextupole windings have been added on another set of 5 narrow quadrupoles [4]. In addition, two symmetrically located sextupoles have been installed in dispersion free regions. As a result, nonlinearities are well controlled and the acceptances found by tracking $\mathrm{A}_{x}=211 \pi \mathrm{mm} \mathrm{mrad}$ and $\mathrm{A}_{y}=188 \pi \mathrm{mm}$ mrad for particles with zero momentum deviation are closed to the values of the linear machine.

Linear coupling produced by the solenoid of the e-cooler becomes stronger at low momenta because the device runs with constant current throughout the cycle. To compensate it, two compensation solenoids have been installed. For perfect coupling compensation, they should be placed as close as possible to the main solenoid. On the other side, the maximum of the acceptance is provided with quadrupoles close to the main solenoid. Due to this, compensation solenoids were placed further apart (Fig. 2). Two skew quadrupoles located symmetrically in other straight sections complete the tools for linear coupling compensation.

\section{MACHINE COMMISSIONING}

\subsection{Injection energy}

With the pre-calculated currents, the $\mathrm{AD}$ has the lower tunes $\mathrm{Q}_{x}=5.33, \mathrm{Q}_{y}=5.29$. To obtain the nominal tunes, about $30 \mathrm{~A}$ were added to all the quadrupoles, that is $1.7 \%$ higher than expected according to magnetization curves measured in the laboratory. After this correction the lat- 


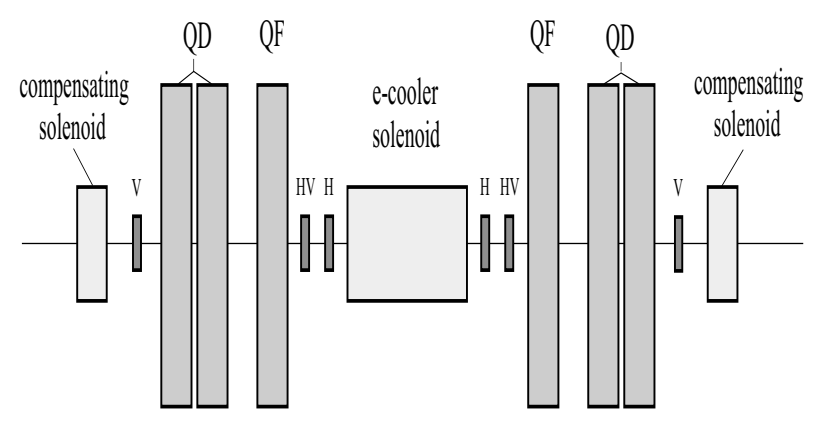

Figure 2: Schematic view of the AD electron cooling insertion.

tice functions at injection energy, found by use of response matrix measurements [5], are fairly close to the theoretical ones. The measured tune dependence on momentum deviation at injection energy is shown in Fig. 3.

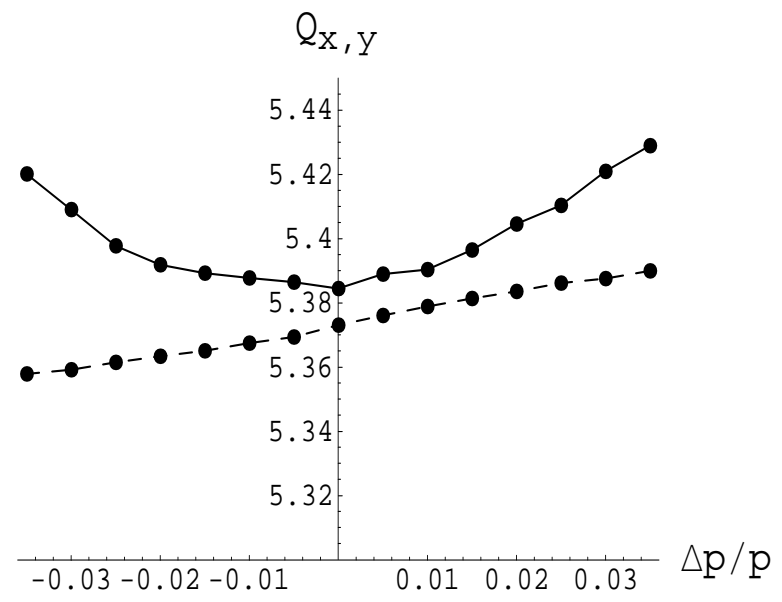

Figure 3: The horizontal and the vertical AD tune at injection energy (solid and dashed line respectively) versus momentum deviation.

The horizontal orbit has been improved in several steps. Some of the orbit excursion comes from a difference between the wide and the narrow bending magnets (that are located in regions with relatively large and small dispersion respectively). This was corrected by a trim supply connected to the wide bending magnets only. Then the orbit was improved by moving a few quadrupoles in the horizontal plane. Finally, local bumps were used to move the orbit inside the elements where optics calculations predict acceptance limitations. For these bumps the dipole correction is available by powering the trim supplies installed on 12 of the 24 bending magnets. For every bump amplitude the acceptance was measured by blowing up the beam, then scraping it. As a result, the best setting for the correctors was defined. The horizontal acceptance was found to be $180 \pi \mathrm{mm}$ mrad, about $15 \%$ less than expected.

In the vertical plane no "global" orbit correction was done. Local bumps were used in a similar way to the horizontal plane except that due to the lack of the verti- cal correctors, machine quadrupoles had to be moved. The measured vertical acceptance was found $200 \pi \mathrm{mm} \mathrm{mrad}$ (Fig. 4), 6\% more than expected from the design.

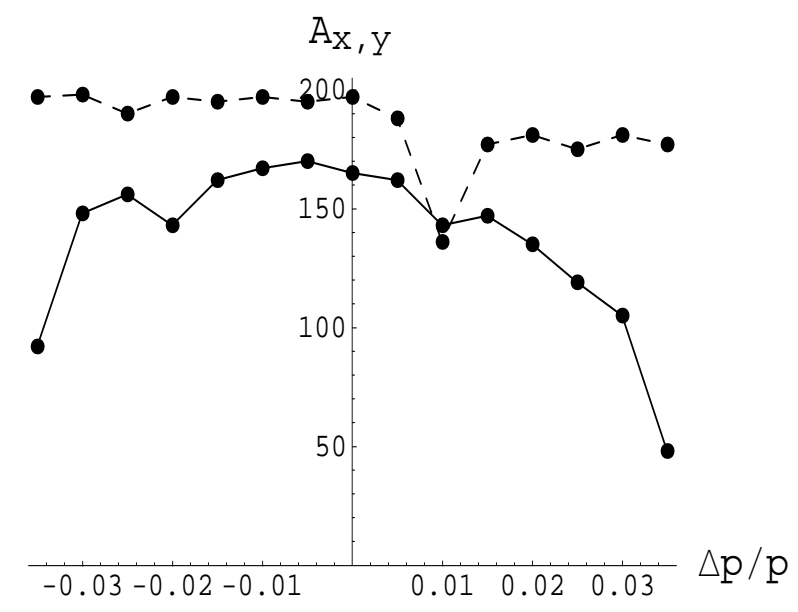

Figure 4: The horizontal and the vertical AD acceptances (solid and dashed line respectively) versus momentum deviation at injection energy. The measurements were done at early stage of the commissioning.

\subsection{Low energy}

During the early commissioning it was found that the beam was decelerated only to $300 \mathrm{MeV} / \mathrm{c}$ and lost at smaller momenta, probably due to tune excursions in the ramp. For this reason the low energy working point was changed to $\mathrm{Q}_{x}=5.45, \mathrm{Q}_{y}=5.42$ (also shown in Fig. 1) to provide more resonance-free room during deceleration. The "Q-jump" is performed at $2 \mathrm{GeV} / \mathrm{c}$ just after stochastic cooling to take advantage of a small beam size for crossing resonance $5 \mathrm{Q}_{x}=27$. The initial working point is kept for injection energy because it provides more acceptance, hence more intensity.

To provide a room for the injection line, two AC quadrupoles QDN53 and QFN54 were manufactured as "half-quadrupoles". Their integrated strengths at injection energy are identical to the other quadrupoles of their families. However during the commissioning it was found by orbit response measurements [5] that this is no longer the case at low momenta and the resulting gradient error disturbs the optics significantly. The QFN54 integrated strength is $12 \%$ less than the other quadrupoles of the family. With the proximity of the horizontal tune to the halfinteger resonance, this produces beta function distortions up to $100 \%$ in the horizontal plane (Fig. 5). In the vertical plane the distortions are smaller, about $25 \%$. To correct the optics at low energy, a separate power supply for QFN54 was installed. The strength of QDN53 is reduced by 3\% w.r.t. other members of that family. In addition, its effect in the horizontal plane is weaker due to a smaller horizontal beta function in the vertically focusing quadrupole, and in 


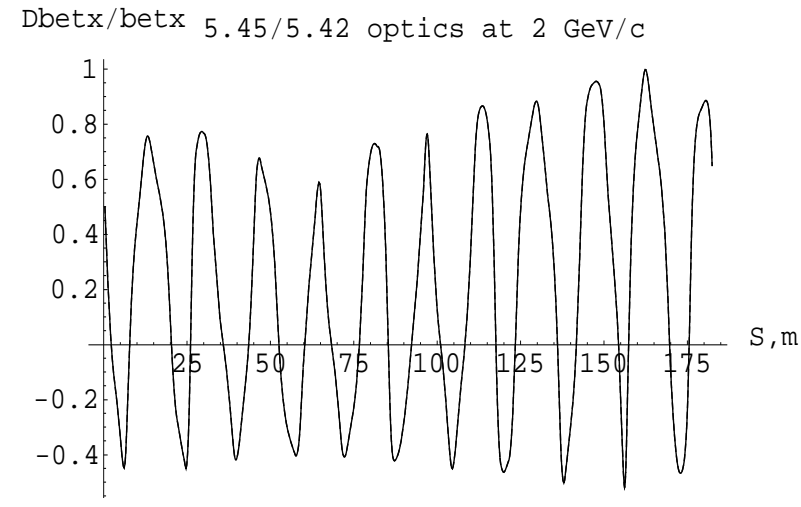

Figure 5: Relative deviations of the horizontal beta function from the designed one due to the gradient errors.

the vertical plane the tune is more distanced from the halfinteger, hence its effect on optics is much less important.

Between injection energy and $2 \mathrm{GeV} / \mathrm{c}$, some of the quadrupoles come out of saturation. To maintain the same optics during the cycle, one could scale the current according to the magnetization curves. But for AD the total number of different families which define the machine optics is 11, while only 7 power supplies are available. Fortunately, this is not critical for machine operation, because the requirements for acceptance are relaxed after beam "compression" by the stochastic cooling at injection energy.

For momenta lower than $2 \mathrm{GeV} / \mathrm{c}$ no magnetization curves are available for some of the quadrupole families. The optics in that range was set up by use of response matrix measurements. With beam intensities above $10^{7}$ the reproducibility of the orbit measurements at $100 \mathrm{MeV} / \mathrm{c}$ (the most difficult case) is about $0.2 \mathrm{~mm}$. To improve the precision of the optics, statistics was accumulated by measuring several response matrices. These measurements can be done in a "parasitic mode" without disturbing machine operation by exciting one orbit at exactly the same moment during every machine cycle.

The response matrix measurements were also extensively used for the calibration of the 4 horizontal orbit correctors located around the electron cooler (see Fig. 2). They are intended for the local compensation of toroid kicks produced by the e-cooler and for the orbit adjustment inside the e-cooler to align the antiprotons w.r.t. the electron beam. Most of the corrector strength is required to compensate the horizontal toroid kicks $(15 \mathrm{mrad}$ at 300 $\mathrm{MeV} / \mathrm{c}$ ). The rest of strength can be used for the beam alignment. Unfortunately due to saturation the correctors are about $45 \%$ weaker than the design values for the inner pair and about $25 \%$ for the outer pair. The modification of the environment around the e-cooler and other schemes for the toroid kick compensation and orbit alignment are being considered.

The linear coupling introduced by the solenoid of ecooler has a strong effect on deceleration efficiency at low momenta. With small residual coupling due to errors and fairly symmetric optics, two power supplies (one for the compensating solenoids and one for the skew quadrupoles) is enough to suppress the difference resonance $\mathrm{Q}_{x}-\mathrm{Q}_{y}=0$ (by minimization of the distance between normal modes) and the sum resonance $\mathrm{Q}_{x}+\mathrm{Q}_{y}=11$ (that is based on the knowledge of the operational optics). After a proper setting of the elements the room in the tune diagram that provides the best beam lifetime was significantly improved.

The stability of the power supplies is crucial for an operation at low momenta. A change in the power supply of a magnetic element leads to an orbit jump that modifies the position and the angle of the extracted beam. To provide a stable ejected beam, permanent monitoring of the machine orbit at low momenta is performed. By comparing the current orbit and the reference one, the source of an orbit jump can be identified and cured. Work is under way to improve the machine stability.

\section{CONCLUSION}

After small hardware modifications and adjustments, caused mainly by the effect of the environment, the AD optics resulted in a good machine performance in the momentum ranged from $3.57 \mathrm{GeV} / \mathrm{c}$ to $100 \mathrm{MeV} / \mathrm{c}$. The machine model is in a good agreement with the design and is extensively used for the machine development and improvement.

\section{REFERENCES}

[1] S. Maury, Commissioning and Operation of the Antiproton Decelerator at CERN (this conference).

[2] P. Beloshitsky e.a., Beam Optics Issues for the Antiproton Decelerator, Proceedings of 1997 Particle Accelerator Conference, Vancouver, v. 2, p. 1382.

[3] B. Autin, Non-linear betatron oscillations, in: CERN Accelerator School, Third Advanced Accelerator Course, p. 1, CERN 90-04, 1990.

[4] P. Beloshitsky and R. Giannini, AD lattice, CERN PS/HP/Note 97-28, September 1997.

[5] P. Beloshitsky and C. Carli, Optics Investigations by Means of Orbit Response Measurements, CERN PS/CA/Note 2000005, 2000; P. Beloshitsky and C. Carli, Considerations on AD Optics Improvement Based on Response Matrix Measurements, CERN PS/OP Note 2000-010, 2000. 